\title{
MICRO-CT IN TISSUE ENGINEERING SCAFFOLDS DESIGNED FOR BONE REGENERATION: PRINCIPLES AND APPLICATION
}

\author{
"MARTIN BARTOŠ*,****,*****, TOMÁŠ SUCHÝ**, ZBYNĚK TONAR***, \\ RENÉ FOLTÁN*, MARIE HUBÁLEK KALBÁČOVÁ**** \\ *Institute of Dental Medicine, First Faculty of Medicine, \\ Charles University and General University Hospital in Prague, Prague 2, Czech Republic \\ **Department of Composites and Carbon Materials, Institute of Rock Structure and Mechanics, \\ Academy of Sciences of the Czech Republic, Prague 8, Czech Republic \\ ***Department of Histology and Embryology and Biomedical Centre, Faculty of Medicine in Pilsen, \\ Charles University, Pilsen, Czech Republic \\ ****Institute of Pathological Physiology, First Faculty of Medicine, Charles University, Prague 2, Czech Republic \\ *****Institute of Anatomy, First Faculty of Medicine, Charles University, Prague 2, Czech Republic \\ "E-mail: martin.bartos@1f1.cuni.cz
}

Submitted December 23, 2017; accepted February 14, 2018

\begin{abstract}
Keywords: Micro-CT, Tissue engineering scaffold, Bone regeneration, Biomaterials, 3D analysis
Micro-CT (micro-computed tomography) is a modern preclinical imaging method allowing non-destructive visualizations and structure analysis yielding at a resolution of a few micrometres. Tissue engineering scaffolds are a promising treatment for bone defects. Micro-CT application for the evaluation of tissue engineering scaffolds for bone surgery and traumatology application is increasing, which comprises in vitro, in vivo and ex vivo studies. Micro-CT itself is not able to replace conventional approaches completely, such as scanning electron microscopy (SEM) or histological examination, but it may offer important benefits regarding non-destructive approach, direct 3D model structure analysis, and visualization and time efficacy. The overview presented herein focuses on a micro-CT application in the field of tissue engineering scaffolds aimed at bone regeneration.
\end{abstract}

\section{THEORETICAL AND DISCUSSION}

Tissue engineering scaffolds and bone regeneration

The application of biomaterials in the form of scaffolds is considered as a promising method for treatment of bone defects resulting from pathological conditions (e.g. trauma, tumour, inflammation) [1] The current gold-standard method is autologous bone grafting $[2,3]$. However, it is limited by the bone defect volume and requires second operating field, which may increase morbidity and the probability of associated complications [4]. The possibility of using artificially produced biomaterials with similar effect on bone healing and predictable outcomes would be advantageous. Scaffolds for bone regeneration are usually porous and biodegradable. Scaffolds must provide temporary three-dimensional support for new bone formation with a desirable biodegradation profile. Various methods for preparation exist (e.g. freeze-drying, solvent casting, electrospinning, rapid prototyping) leading to different 3D structure $[5,6]$. Scaffolds are frequently based on natural or synthetic polymers (e.g. collagen, polylactide) in combination with hydroxyapatite par- ticles [7]. Collagen is frequently cross-linked to improve its chemical and mechanical properties $[8,9]$. Such scaffolds should present appropriate chemical, biological and mechanical properties that are necessary to promote normal cellular behaviour and function [10]. A major challenge remains in terms of designing an ideal bone graft that mimics the features of natural bone, both in terms of the main composition and hierarchical microstructure [11]. Biologically active substances can be incorporated into scaffold structure to enhance its effect (e.g. BPM - bone morphometric proteins; [12]) and cell seeding (e.g. mesenchymal stem cells) can be performed prior to implantation [13].

\section{Scaffold structure - its importance and methods of analysis}

Three-dimensional scaffold structure is considered to have major influence on scaffold-tissue interaction, especially when considering porosity arrangement. 3D structure is usually very complex and complicated across scales. Prevalence of open pores with a high degree of interconnectivity and convenient pore sizes substantially influences cell migration, vascularisation, 
fluid flow, mechanical properties, and available surface [14]. Pore sizes ranging from 100 to $300 \mu \mathrm{m}$ were found to be the most efficient for new bone formation $[15,16]$. However, pore size values are dependent on the method of measurement (our unpublished results) and no consent in this respect exists. Scaffold structure is usually evaluated in dry state, which may not be accurate, as implantation into the bone defect inevitably leads to scaffold hydration, which can change the threedimensional structure due to hydrophilic compounds.

Scaffold structure analysis is achievable by numerous methods. Total porosity can be assessed e.g. by using gravimetry. Open porosity can be measured by liquid displacement or mercury intrusion [17, 18]. In some scaffolds, the application of these methods may alter its structure. Liquid displacement may be influenced by scaffold swelling due to hydrophilic compounds. A common approach is scanning electron microscopy (SEM) image analysis, which allows the measurement of various structural parameters (e.g. pore diameters, area, shape, wall and struts thickness). SEM image analysis offers precise visualization, is readily available and affordable. However, there are many drawbacks. Based on sectioning, this approach is destructive, time consuming, orientation dependant and limited to two-dimensions. 3D structure evaluation is achievable by using the stereology method based on structure assumptions. Evaluation of pore size based on a $2 \mathrm{D}$ section may not be accurate because the designation of scaffold walls is often very unclear (it may not be evident, whether the structure is directly on or below the section plane). Differentiating between closed and open pores from SEM images is unreachable, and alternative methods (e.g. mercury intrusion porositometry) may alter the scaffold structure, as mentioned above.

\section{Micro-CT analysis of scaffold structure}

\section{Basic principles}

Micro-CT (microtomography) is an X-ray preclinical imaging method, which enables both $2 \mathrm{D}$ and $3 \mathrm{D}$ structure analysis and visualization $[19,20]$. Important benefits are non-destructivity, time efficacy and direct 3D model analysis. Isotropic pixel or voxel size in modern micro-CT devices is below $1 \mu \mathrm{m}$, but continuous improvement of resolution in micro-CT devices has to be considered, especially when evaluating previous studies. Its employment in the field of tissue engineering is still increasing, especially in the structure characterization of biomaterials. However, its availability is still limited because of its high acquisition price.

Specimens are scanned in micro-CT, usually in air or in a plastic tube, placed on a microstage inside a device. Appropriate specimen size differs according to the microCT device. In ex vivo desktop micro-CT designed for material testing the usual specimen size is $0.1-10 \mathrm{~cm}^{3}$, while in in vivo devices, specimen size can be larger, enabling live mice or rats can be subjected to scanning. Scanning parameters comprise of resolution (pixel size), camera binning, source, current, filter, rotation step, $180^{\circ}$ or $360^{\circ}$ rotation etc. Image acquisition leads to a dataset of projection images, which are reconstructed into a $2 \mathrm{D}$ cross-section greyscale image dataset (usually based on a modified Feldkamp algorithm; Figure 1). Image data can be subjected to further modifications (e.g. creating regions or volumes of interest) and image processing (e.g. noise reduction, morphological operations, binary operations). Visualizations may be achieved using 2D virtual sections or $3 \mathrm{D}$ visualizations created by volume or surface rendering. Computed tomography generally leads to the presence of specific artifacts in image data, such as ring artifacts, beam hardening or misalignment and the presence of image noise, which may negatively influence visualizations and analyses [21].

\section{Micro-CT analysis}

Prior to analysis, image data must be converted from greyscale to black and white pixels (binarization), so the object (white colour) and background (black colour) are clearly differentiated. This process may significantly influence the results of the following analysis. The influence depends on the structure of the analysed object (simple or complex), material composition, homogeneity and image data quality (e.g. image noise, artifacts). The influence of image binarization on the structural parameters was evaluated for bone tissue $[22,23]$. However, the binarization effect in scaffolds is yet to be sufficiently studied, and is complicated by the overlapping X-ray density of its materials, thin structures and partial volume effect [24, 25].

Micro-CT enables the evaluation of many structural 2D and 3D parameters. Micro-CT offers 2D analysis based on individual sections (slices) or direct 3D model analysis, which is orientation independent. Basic parameters are e.g. specimen volume, surface, intersection surface, number of objects and porosity (open and closed pores). Volume and surface parameters are measured using a marching-cubes algorithm. Structure thickness and separation is calculated based on a medial axis computation and sphere-fitting algorithm, which offers new insight into structure evaluation in $3 \mathrm{D}$, and may be applied for pore size measurement [26, 27, 28]. Many other parameters may be obtained as a result of 3D analysis e.g. anisotropy, Euler number, fractal index and Structure Model Index [20, 26]. However, certain parameters provided by micro-CT analytical software must be used with caution, since their interpretation is not straightforward in such complex structures. Image processing and analysis can be partially automated leading to better time efficacy, and a reduction of subjectivity in evaluation. Micro-CT visualizations prior to histological examination may improve sectioning orientation thus 
improving its outcomes. Together with its advantages, micro-CT presents important drawbacks: limitation by resolution (even though modern devices offer pixel or voxel size below $1 \mu \mathrm{m}$, it is still substantially less than e.g. in SEM) and high-dependency on image data and its processing prior to analysis.

Micro-CT application: in vitro, in vivo, ex vivo

\section{In vitro}

Tissue engineering scaffold structure is usually studied in dry state and micro-CT may be a complementary method to conventional analysis $[15,17,29]$. The fabrication process and its modifications can be evaluated and thus optimized with regard to appropriate structural parameters (e.g. total porosity, open porosity, pore size). Based on micro-CT results, the most prospective types of fabricated scaffolds can be selected for further evaluation. Structural changes related to hydration are considered very important, but their assessment is difficult to reach. Micro-CT is able to provide such analysis. However, only a few micro-CT studies focus on the evaluation of both hydrated and dry-state scaffolds to date $[30,31]$. There are technical difficulties resulting from scanning hydrated scaffolds, e.g. insufficient X-ray contrast between scaffold matrix and solution or motion artifacts caused by gravitation, specimen rotation and swelling. X-ray contrast has to be enhanced by contrast agents (e.g. phosphotungstic acid or iodine solution;

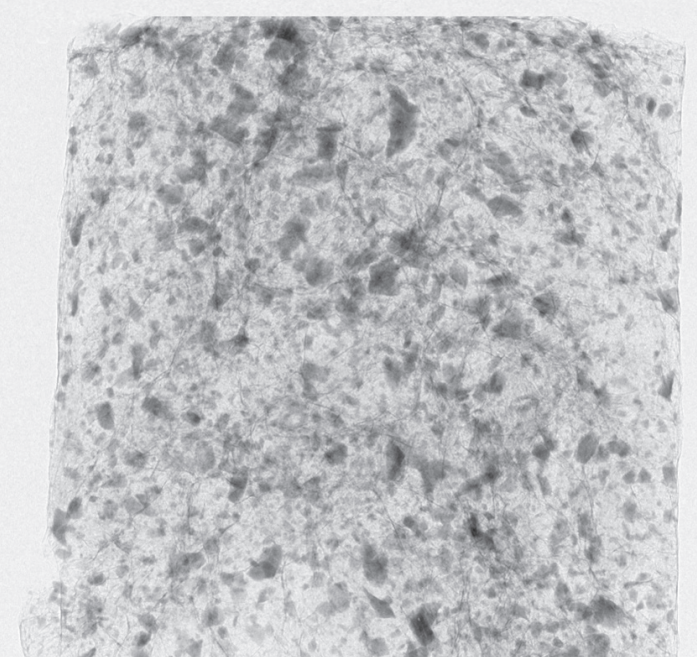

a)

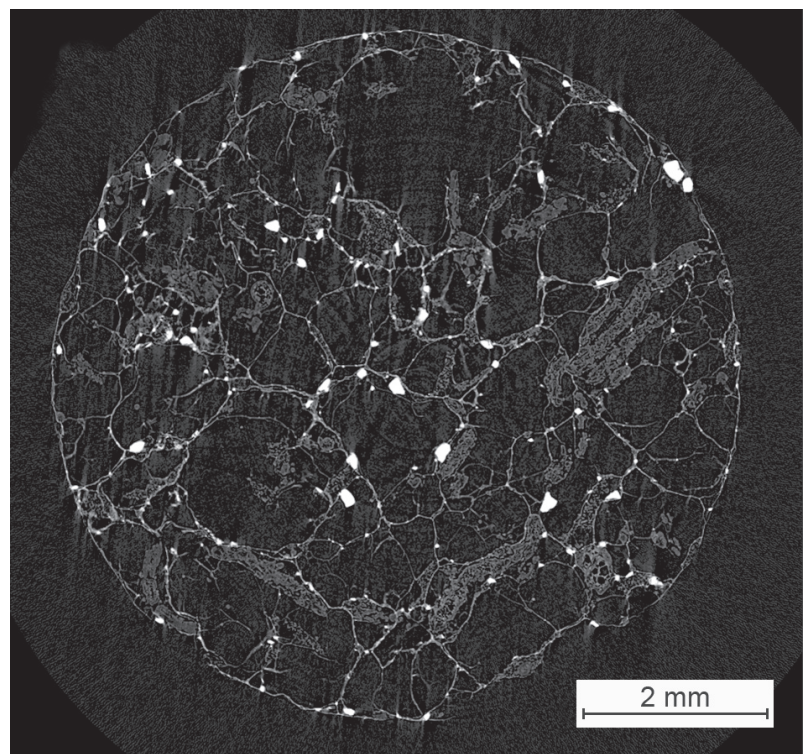

c)

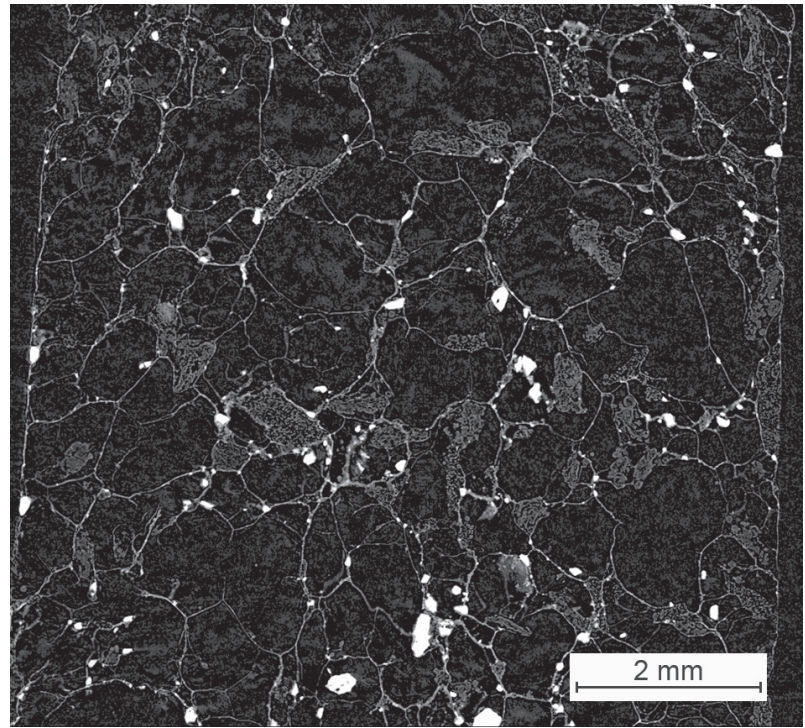

b)

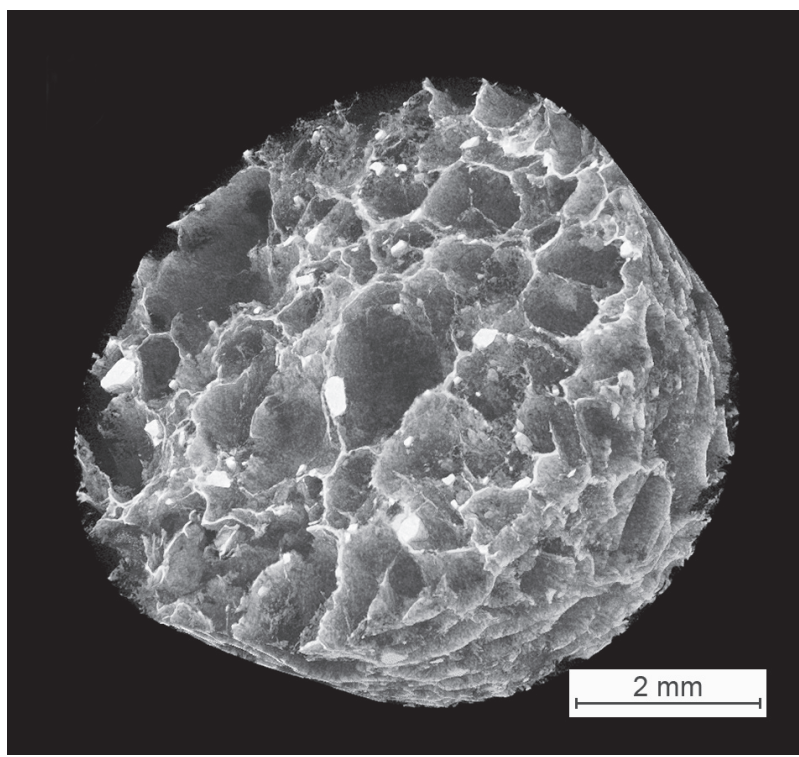

d)

Figure 1. Illustration of micro-CT scanning of collagen-based scaffold using desktop micro-CT SkyScan 1272 with $4 \mu \mathrm{m}$ pixel size (Bruker, Belgium): a) 2D projection image, b, c) reconstructed 2D cross-section images d) 3D visualization. 
[32]). However, its influence on scaffold properties remains relatively unknown, and therefore may lead to alterations.

Cell seeding (e.g. by mesenchymal stem cells) prior to implantation may be performed in order to improve the effect on bone healing and regeneration $[13,9,33]$. Assessment of the presence and distribution of seeded cells in the whole scaffold in 3D would be advantageous, otherwise it is limited to specimen sectioning. Only a few studies have focused on cell visualization [34]. Cells have to be X-ray contrasted to provide appropriate contrast. However, this is not standard application since the resolution of even modern devices is insufficient (or nearly insufficient) regarding cell size in $3 \mathrm{D}$, and cell to scaffold X-ray contrast. Nanocomputed tomography (nano-CT), a new developing field of preclinical imaging, may introduce cells and scaffold scanning as a common procedure.

\section{In vivo and ex vivo}

Micro-CT can be employed for evaluation of implantation of tissue engineering scaffold into bone defect. There are two basic approaches: in vivo and ex vivo [35]. The first uses an in vivo micro-CT device capable of scanning of live animals (usually mice or rats), which

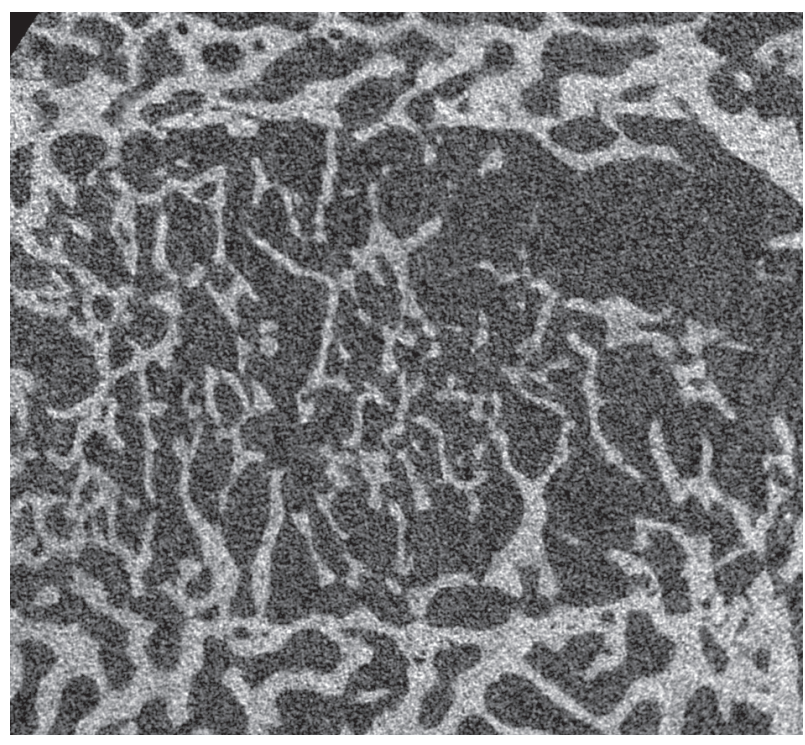

a)

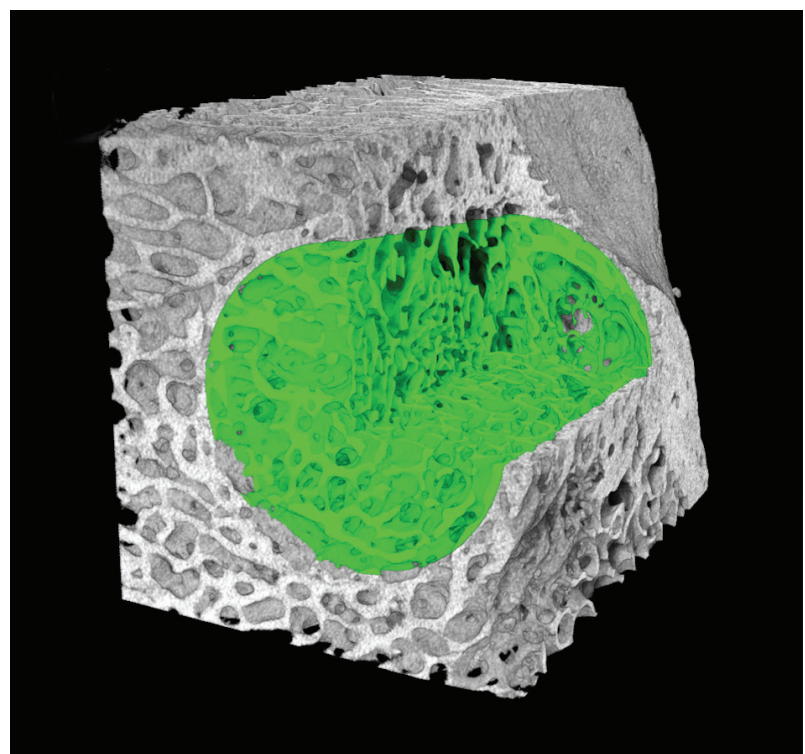

c)

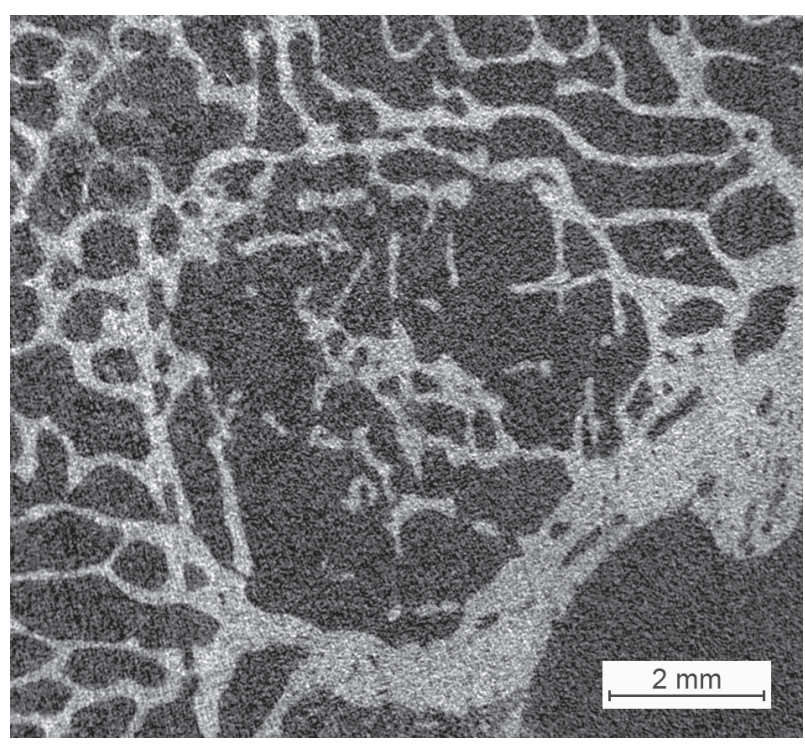

b)

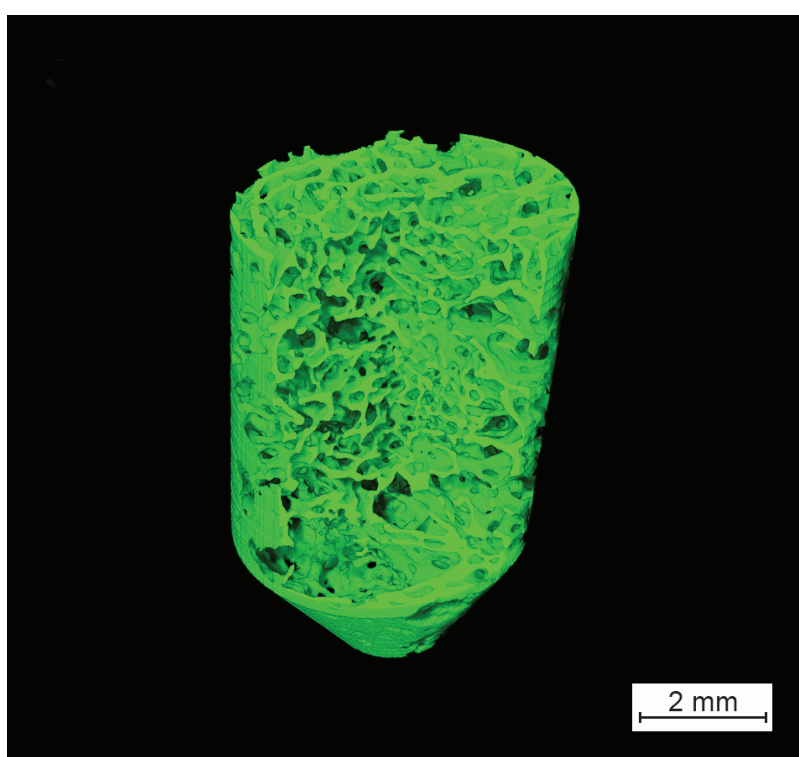

d)

Figure 2. Demonstration of micro-CT 2D and 3D visualizations of bone defect treated by autologous bone graft. Images from preliminary ex vivo study: a, b) 2D cross sections: former bone defect is evident, but new bone tissue formation is clearly observed, c) volume of former bone defect is depicted with new bone tissue d) segmented new bone tissue, which may be subjected to 3D analysis. 
are anesthetized and their vital functions are controlled. This method is well established for studying metabolic diseases influencing bone tissue (e.g. osteoporosis), fracture healing, bone tissue engineering or tumour bone metastases [36, 37, 38, 39]. The evaluation of the same specimen at a different time enables both the precise observation of newly generated bone tissue and scaffold material degradation. This leads to a higher efficiency of such experiments based on an increasing number of measurements. Moreover, the number animals experimented on can be reduced, which is advantageous from the ethical point of view and leads to financial cost reduction. Repeated scanning of the same animal does not result in the alteration of bone structure and its healing processes [40, 41]. In vivo scans are much faster (minutes) compared to ex vivo scans (hours). However, resolution is lower compared to ex vivo devices that may negatively influence the evaluation of thin bone structures and in particular scaffold structure. Since micro-CT detects only X-ray dense objects, differentiation between new bone and scaffold structure is based mainly on subjective assessment of visualized structure and may not be evident. The detection of less mineralized bone and osteoid is not sufficient, so verification by histological examination is appropriate. Ex vivo micro-CT analysis combines benefits of nondestructive high-resolution analysis and scaffold-tissue interactions (Figure 2). Specimens are harvested after the euthanization of the experimental animal in a defined time interval after implantation. Volume of interest (VOI) is defined in selected specimen (usually in the place of former bone defect), so effect of different treatment approaches may be compared (e.g. autologous bone graft versus tissue engineering scaffold implantation). Visualization of VOI in 3D and virtual 2D sections in any selected plane helps understanding to bone regeneration and scaffold degradation process. Volume of new bone, its structure, homogeneity and spatial distribution (e.g. peripheral versus central regions) is considered.

\section{CONCLUSION}

Micro-CT is an important preclinical imaging method for structure analysis and visualization. Its application in the field of tissue engineering is notably increasing. Promising bone defect treatments comprise of tissue-engineering scaffolds. Their 3D structure is of great importance and micro-CT can be employed in different settings (in vitro, in vivo and ex vivo) for its evaluation. Major advantages are non-destructivity, direct 3D model analysis and time efficiency. In cases following histological examinations, micro-CT improves the orientation of specimen sectioning. The major disadvantage of micro-CT is a high dependency on image data processing, which is generally not standardized. The overview presented herein focuses on micro-CT application in bone engineering scaffolds.
Acknowledgements

This study was supported by the Ministry of Education, Youth and Sports (Progress Q29/LF1), Ministry of Health of the Czech Republic (NV 15-25813A) and Grant Agency of Czech Republic (Reg. No. 1437368G). Supported by "Technological development of post-doc programs" project (registration number CZ.1.05/41.00/16.0346) supported by the Research and Development for Innovations Operational Programme (RDIOP), co-financed by European regional development funds and the state budget of the Czech Republic.

\section{REFERENCE}

1. Billström G. H., Blom A. W., Larsson S., Beswick A. D. (2013): Application of scaffolds for bone regeneration strategies: current trends and future directions. Injury, 44, S28-S33. doi: 10.1016/S0020-1383(13)70007-X

2. Pape H. C., Evans A., Kobbe P. (2010): Autologous bone graft: properties and techniques. Journal of Orthopaedic Trauma, 24, S36-S40. doi: 10.1097/BOT.0b013e3181cec4a1

3. Ferretti C., Muthray E., Rikhotso E., Reyneke J., Ripamonti U. (2016): Reconstruction of 56 mandibular defects with autologous compressed particulate corticocancellous bone grafts. British Journal of Oral and Maxillofacial Surgery, 54(3), 322-326. doi: 10.1016/j.bjoms.2015.12.014

4. Dimitriou R., Mataliotakis G. I., Angoules A. G., Kanakaris N. K., Giannoudis P. V. (2011): Complications following autologous bone graft harvesting from the iliac crest and using the RIA: a systematic review. Injury, 42, S3-S15. doi: 10.1016/j.injury.2011.06.015

5. Sultana N., Hassan M. I., Lim M. M. (2015): Composite Synthetic Scaffolds for Tissue Engineering and Regenerative Medicine. Springer. doi: 10.1007/978-3-319-09755-8_2

6. Gregor A., Filová E., Novák M., Kronek J., Chlup H., Buzgo M., Blahnová V., Lukášová V., Bartoš M., Nečas A., Hošek J. (2017): Designing of PLA scaffolds for bone tissue replacement fabricated by ordinary commercial $3 \mathrm{D}$ printer. Journal of Biological Engineering, 11(1), 31. doi:10.1186/ s13036-017-0074-3

7. Gloria A., Santis R., Ambrosio L. (2010): Polymer-based composite scaffolds for tissue engineering. Journal of Applied Biomaterials \& Biomechanics, 8(2), 57-67.

8. Liu X., Ma P. X. (2004): Polymeric scaffolds for bone tissue engineering. Annals of Biomedical Engineering, 32(3), 477-486. doi:10.1023/B:ABME.0000017544.36001.8e

9. Suchý T., Šupová M., Sauerová P., Verdánová M., Sucharda Z., Rýglová Š., Žaloudková M., Sedláček R., Kalbáčová M. H. (2015): The effects of different cross-linking conditions on collagen-based nanocomposite scaffolds - an in vitro evaluation using mesenchymal stem cells. Biome-dical Materials, 10(6), 065008. doi: 10.1088/1748-6041/10/6/ 065008

10. Polo-Corrales L., Latorre-Esteves M., Ramirez-Vick J. E. (2014): Scaffold design for bone regeneration. Journal of Nanoscience and Nanotechnology, 14(1), 15-56. doi: 10.1166/jnn.2014.9127

11. Liu W. F., Chen C. S. (2005): Engineering biomaterials to control cell function. Materials Today, 8(12), 28-35. doi: 10.1016/S1369-7021(05)71222-0 
12. Blokhuis T. J., Lindner T. (2008): Allograft and bone morphogenetic proteins: an overview. Injury, 39, S33-S36. doi: 10.1016/S0020-1383(08)70013-5

13. Tollemar V., Collier Z. J., Mohammed M. K., Lee M. J., Ameer G. A., Reid R. R. (2016): Stem cells, growth factors and scaffolds in craniofacial regenerative medicine. Genes \& Diseases, 3(1), 56-71. doi: 10.1016/j.gendis.2015.09.004

14. Mastrogiacomo M., Scaglione S., Martinetti R., Dolcini L., Beltrame F., Cancedda R., Quarto R. (2006): Role of scaffold internal structure on in vivo bone formation in macroporous calcium phosphate bioceramics. Biomaterials, 27, 3230-3237. doi: 10.1016/j.biomaterials.2006.01.031

15. Karageorgiou V., Kaplan D. (2005): Porosity of 3D biomaterial scaffolds and osteogenesis. Biomaterials, 26(27), 5474-5491. doi: 10.1016/j.biomaterials.2005.02.002

16. Gomez S., Vlad M. D., López J., Fernández E. (2016): Design and properties of 3D scaffolds for bone tissue engineering. Acta Biomaterialia, 42, 341-350. doi: 10.1016/ j.actbio.2016.06.032

17. Ho S. T., Hutmacher D. W. (2006): A comparison of micro CT with other techniques used in the characterization of scaffolds. Biomaterials, 27(8), 1362-1376. doi: 10.1016/j. biomaterials.2005.08.035

18. Jones A. C., Arns C. H., Sheppard A. P., Hutmacher D. W., Milthorpe B. K., Knackstedt M. A. (2007): Assessment of bone ingrowth into porous biomaterials using MICRO-CT. Biomaterials, 28(15), 2491-2504. doi: 10.1016/j.biomaterials.2007.01.046

19. Landis E.N., Keane D.T., (2010): X-ray microtomography. Materials Characterization, 61, 1305-1316. doi: 10.1016/j. matchar.2010.09.012

20. Schladitz K. (2011): Quantitative micro-CT. Journal of Microscopy, 243(2), 111-117.

doi: 10.1111/j.1365-2818.2011.03513.x.

21. Boas F. E., Fleischmann D. (2012): CT artifacts: causes and reduction techniques. Imaging Med, 4(2), 229-240. doi: 10.2217/iim.12.13

22. Yan Y. B., Qi W., Qiu T. X., Teo E. C., Lei, W. (2012): The effect of threshold value on the architectural parameters and stiffness of human cancellous bone in micro ct analysis. Journal of Mechanics in Medicine and Biology, 12(05), 1250092. doi: 10.1142/S0219519412500923

23. Hara T., Tanck E., Homminga J., Huiskes, R. (2002): The influence of microcomputed tomography threshold variations on the assessment of structural and mechanical trabecular bone properties. Bone, 31(1), 107-109.

24. Zidek J., Vojtova L., Abdel-Mohsen A. M., Chmelik J., Zikmund T., Brtnikova J., Roman Jakubicek R., Zubal L., Jan J., Kaiser, J. (2016): Accurate micro-computed tomography imaging of pore spaces in collagen-based scaffold. Journal of Materials Science: Materials in Medicine, 27(6), 110. doi: 10.1007/s10856-016-5717-2

25. Stock S.S. (2009). MicroComputed Tomography: Methodology and Applications. CRC Press.

26. Bruker micro-CT, Morphometric parameters measured by Skyscan CT-analyser software

27. Hildebrand T., Rüegsegger P. (1997): A new method for the model-independent assessment of thickness in threedimensional images. Journal of Microscopy, 185(1), 67-75. doi: 10.1046/j.1365-2818.1997.1340694.x

28. Rémy É., Thiel É. (2002): Medial axis for chamfer distances: computing look-up tables and neighbourhoods in 2D or 3D. Pattern Recognition Letters, 23(6), 649-661. doi: 10.1016/S0167-8655(01)00141-6
29. Peyrin F. (2011): Evaluation of bone scaffolds by microCT. Osteoporosis International, 22(6), 2043-2048. doi: 10.1007/s00198-011-1609-y

30. Offeddu G. S., Ashworth J. C., Cameron R. E., Oyen M. L. (2016): Structural determinants of hydration, mechanics and fluid flow in freeze-dried collagen scaffolds. Acta Biomaterialia, 41, 193-203. doi: 10.1016/j.actbio.2016.05.024

31. Suchý T., Šupová M., Bartoš M., Sedláček R., Piola M., Soncini M., Fiore G.B., Sauerová P., Kalbáčová M. H. (2018): Dry versus hydrated collagen scaffolds: are dry states representative of hydrated states? Journal of Materials Science: Materials in Medicine, 29(3), 20. doi: 10.1007/ s10856-017-6024-2

32. Metscher B. D. (2009): MicroCT for comparative morphology: simple staining methods allow high-contrast 3D imaging of diverse non-mineralized animal tissues. $B M C$ physiology, 9(1), 11. doi:10.1186/1472-6793-9-11

33. Sundelacruz S., Kaplan D. L. (2009). Stem cell-and scaffoldbased tissue engineering approaches to osteochondral regenerative medicine. In Seminars in cell \& developmental biology (Vol. 20, No. 6, pp. 646-655). Academic Press. doi: 10.1016/j.semcdb.2009.03.017

34. Farini A., Villa C., Belicchi M., Meregalli M., Torrente Y. (2013). Micro-CT technique for three-dimensional visualization of human stem cells. In Imaging and Tracking Stem Cells (pp. 143-152). Humana Press, Totowa, NJ. doi: 10.1007/7651_2013_23

35. Campbell G. M., Sophocleous A. (2014): Quantitative analysis of bone and soft tissue by micro-computed tomography: applications to ex vivo and in vivo studies. BoneKEy reports, 3. doi: 10.1038/bonekey.2014.59

36. Umoh J. U., Sampaio A. V., Welch I., Pitelka V., Goldberg H. A., Underhill T. M., Holdsworth D. W. (2009): In vivo micro-CT analysis of bone remodeling in a rat calvarial defect model. Physics in Medicine \& Biology, 54(7), 2147. doi: 10.1088/0031-9155/54/7/020

37. El-Rashidy A. A., Roether J. A., Harhaus L., Kneser U., Boccaccini A. R. (2017): Regenerating bone with bioactive glass scaffolds: A review of in vivo studies in bone defect models. Acta Biomaterialia, 62, 1-28. doi:10.1016/j.actbio. 2017.08.030

38. Johnson L. C., Johnson R. W., Munoz S. A., Mundy G. R., Peterson T. E., Sterling J. A. (2011): Longitudinal live animal micro-CT allows for quantitative analysis of tumorinduced bone destruction. Bone, 48(1), 141-151.

39. Buie H. R., Bosma N. A., Downey C. M., Jirik F. R., Boyd S. K. (2013): Micro-CT evaluation of bone defects: applications to osteolytic bone metastases, bone cysts, and fracture. Medical Engineering and Physics, 35(11), 16451650. doi:10.1016/j.medengphy.2013.05.016

40. Sacco S. M., Saint C., Longo A. B., Wakefield C. B., Salmon P. L., LeBlanc, P. J., Ward W. E. (2017): Repeated irradiation from micro-computed tomography scanning at 2, 4 and 6 months of age does not induce damage to tibial bone microstructure in male and female CD-1 mice. BoneKEy reports, 6. doi:10.1038/bonekey.2016.87

41. Brouwers J. E., Van Rietbergen B., Huiskes R. (2007): No effects of in vivo micro-CT radiation on structural parameters and bone marrow cells in proximal tibia of wistar rats detected after eight weekly scans. Journal of Orthopaedic Research, 25(10), 1325-1332. doi:10.1002/ jor.20439 